\title{
Practical RAKE receiver architecture for the downlink communications in a DS-CDMA mobile system
}

\author{
E. Del Re \\ R. Fantacci \\ P. Giannoccaro
}

Indexing terms: CDMA communication systems, Fading multipath channels, RAKE receivers

\begin{abstract}
The authors deal with a practical architecture for a RAKE receiver suitable for the downlink communication in a direct-sequence code division multiple access (DS-CDMA) mobile system. The proposed RAKE receiver makes use of a pilot signal broadcast by the base station to obtain channel parameter estimation and synchronisation. Computer simulations have been carried out in deriving the performance in terms of bit-error probability under typical multipath fading propagation conditions of GSM applications. Comparisons with an ideal RAKE receiver and with a multipath rejection receiver recently proposed in the literature, for the same type of downlink channel, are shown to highlight the good behaviour of the proposed receiver.
\end{abstract}

\section{Introduction}

Direct-sequence (DS) CDMA seems to be a promising approach for implementing cellular communications services, such as personal mobile communications and indoor wireless networks [1]. A large body of literature exists on the analysis of DS-CDMA cellular systems operating in fading multipath environments [2, 3]. Most analyses of spread-spectrum communications in fading multipath environments assume that the receiver attempts to demodulate the signal received over one fixed path only $[4,5]$. Signals coming from other paths are suppressed, and as a whole considered as an additive white Gaussian noise (AWGN) [5].

A more efficient approach is given by the RAKE receiver first proposed by Price and Green [6]. The effectiveness of the RAKE receiver for detection of digital signals transmitted over a frequency-selective slowly fading channel has been widely proved in the literature [6]. However, the practical implementation of a RAKE receiver is still an unsolved problem. This paper deals with this matter, and in particular, a practical RAKE receiver is proposed, based on an efficient tracking strategy for the estimation of the main channel parameters:

C IEE. 1998

IEE Proceedings online no. 19982135

Paper first received 14th April and in revised form 6th November 1997

The authors are with the Dipartimento di Ingegneria Elettronica Università di Firenze, Via S. Marta 3, 50139 Firenze, Italy where

$$
\left\{\alpha_{k}(t), \vartheta_{k}(t), \tau_{k}(t)\right\} \quad k=1, \ldots, L
$$

$\alpha_{k}(t)=$ attenuation introduced by the $k$-path

$\vartheta_{k}(t)=$ phase shift introduced by the $k$-path

$\tau_{k}(t)=$ time delay introduced by the $k$-path

$L \quad=$ number of resolvable paths

The estimate of the channel parameters $\left\{\alpha_{k}(t), \vartheta_{k}(t)\right.$, $\left.\tau_{k}(t)\right\}$ is obtained by means of a pilot signal broadcast by the base station to all the mobile users. For the sake of simplicity, we will avoid explicity indicating the dependence of the channel parameters on the variable $t$. At the receiver end, a maximum ratio combining approach [7] implemented by means of a RAKE receiver is assumed to collect the signal energy from all the $L$ paths that carry the same information. Owing to the difficulties in deriving the bit-error rate performance of such a (RAKE) receiver, computer simulations have been carried out under fading multipath propagation conditions which are typical of an urban environment.

\section{Communication system and channel model}

The communication system under consideration is formed by a single isolated circular cell of radius $R$ with a centrally located base station. In particular, the focus is on downlink communications. It is assumed that the base station communicates with the $K$ spatially dispersed mobile users via a DS-CDMA scheme. All the $K$ transmitted signals have experienced identical fading when received by a particular mobile user. These users are assumed to be uniformly distributed throughout the cell area. In mobile communication channels, the downlink channel (as well as the uplink channel) is a randomly time-varying channel. A widely used model for randomly time-varying channels is the wide-sensestationary uncorrelated-scattering (WSSUS) channel model [8], shown in Fig. 1. In such a model, the received signal $r(t)$ is given by the sum of the output of a linear system with a randomly time-varying impulse response $g(t, \xi)$, with an AWGN term $n(t)$ with a twosided power spectral density $N_{0} / 2$. The random process $g(t, \xi)$ is assumed to have zero-mean Gaussian statistics independent of $t$. Its autocorrelation function has the form

$$
\begin{aligned}
R_{g}(\tau, \xi, \eta) & =E\left[g(t+\tau, \xi) \cdot g^{*}(t, \eta)\right] \\
& =Q(\tau, \xi) \cdot \delta(\xi-\eta)
\end{aligned}
$$

where ( $)^{*}$ is the complex conjugate function, $Q(\tau, \xi)$ is the delay cross-power density associated with the 
WSSUS channel, and $\delta(t)$ is a function which takes the value 1 for $t$ equal to 0 , and 0 , otherwise.

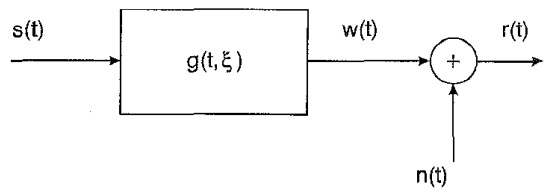

Fig. 1 The WSSUS channel model

The function $Q(\tau) \equiv Q(\tau, 0)$ is called the multipath intensity profile (MIP), and gives the average power output of the communication channel as a function of the time delay $\tau$. A mobile radio channel is often well characterised by an exponential MIP [9]. In particular, this model was the outcome of the European COST207 multipath propagation study (subsequently included in the GSM system specifications [10]). In this paper, time-discrete pseudo-noise code (PN) sequences are considered to obtain a wideband signal. Under certain assumptions [11], the WSSUS channel can be modelled as a tapped delay line. The multipath effects are represented as a sequence of replicas of the transmitted signal, each one characterised by a particular value of the delay, phase and attenuation. The low-pass impulse response of the channel is equal to

$$
h(t)=\sum_{i=1}^{L} \alpha_{i}(t) \delta\left(t-\tau_{i}\right) e^{j \vartheta_{i}(t)}
$$

The terms $\alpha_{i}(t)$ and $\vartheta_{i}(t)$ are stochastic processes, the first is characterised by a Rayleigh distribution, the second uniformly distributed in the $[0,2 \pi)$ interval. Let $T_{\max }$ be the total multipath spread, then we have

$$
L \leq\left\lfloor\frac{T_{\max }}{T_{c}}\right\rfloor+1
$$

where $\lfloor x\rfloor$ is the largest integer contained in $x$. We have also assumed the intersymbol interference introduced by the downlink channel to be negligible. This means that

$$
\tau_{i}<T_{b} \quad i=1, \ldots, L
$$

where $T_{b}$ is the bit duration.

\section{Practical digital detection scheme}

In our communication system, we have assumed that a QPSK modulation scheme is used in transmission. The base-band representation of the in-phase and quadrature components of the signal to be transmitted is

$$
v(t)=\sum_{n=0}^{+\infty}\left(b_{n}+j d_{n}\right) x\left(t-n T_{b}\right)
$$

where

$\left\{b_{n}\right\}=$ in-phase data sequence, with $b_{n}$ equal to \pm 1 with equal probability

$\left\{d_{n}\right\}=$ quadrature data sequence, with $d_{n}$ equal to \pm 1 with equal probability and

$$
x(t)= \begin{cases}1 & 0 \leq t<T_{b} \\ 0 & \text { otherwise }\end{cases}
$$

Each informative component is multiplied by the following PN sequences:

$$
g_{I}(t)=\sum_{n=0}^{M-1} c_{n}^{(I)} p\left(t-n T_{c}\right)
$$

$$
g_{Q}(t)=\sum_{n=0}^{M-1} c_{n}^{(Q)} p\left(t-n T_{c}\right)
$$

where

$\left\{c_{n}^{(l)}\right\}=\mathrm{PN}$ sequence associated with the in-phase component, with $c_{n}^{(I)}$ equal to \pm 1 (binary chip)

$\left\{c_{n}^{(Q)}\right\}=$ PN sequence associated with the quadrature component, with $c_{n}^{(Q)}$ equal to \pm 1 (binary chip)

$T_{c} \quad=$ chip duration

$M=$ number of chips in the PN sequence (or chips/ bit) and

$$
p(t)= \begin{cases}1 & 0 \leq t<T_{c} \\ 0 & \text { otherwise }\end{cases}
$$

A further PN pilot sequence is added to the transmitted signal to accomplish the channel parameters estimation at each receiving end. This pilot sequence is

$$
g_{E}(t)=\sum_{n=0}^{M-1} c_{n}^{(E)} p\left(t-n T_{c}\right)
$$

where $c_{n}^{(E)}= \pm 1$ denotes the binary chips forming the $\mathrm{PN}$ sequence.

The use of a pilot signal broadcast by the base station to derive the channel parameter estimation has been previously proposed by Viterbi in [12]. Differently from [12], here the pilot sequence is added to each user sequence. Moreover, we derive results for a specific channel model, which is of wide interest in GSM applications.

According to the transmission scheme sketched in Fig. 2, the transmitted signal is

$$
\begin{aligned}
& s(t) \\
& =\sum_{n=0}^{+\infty} \sum_{k=0}^{M-1}\left(b_{n} c_{k}^{(I)}+j d_{n} c_{k}^{(Q)}\right) p\left(t-k T_{c}-n T_{b}\right) \\
& \quad+\sum_{n=0}^{+\infty} \sum_{k=0}^{M-1} c_{k}^{(E)} p\left(t-k T_{c}-n T_{b}\right)
\end{aligned}
$$

From eqn. 3 , if only one active user is assumed, it is a straightforward matter to see that the received signal is

$$
\begin{aligned}
& r(t)= \sum_{n=0}^{+\infty} \sum_{k=0}^{M-1} \sum_{i=1}^{L} \alpha_{i}(t)\left[\left(b_{n} c_{k}^{(I)}+j d_{n} c_{k}^{(Q)}\right)\right. \\
&+\sum_{n=0}^{+\infty} \sum_{k=0}^{M-1} \sum_{i=1}^{L} \alpha_{i}(t) c_{k}^{(E)} \\
& \cdot p\left(t-k T_{c}-n T_{b}-\tau_{i}\right) e^{j \vartheta_{i}(t)}+n(t)
\end{aligned}
$$

The digital detection scheme considered in this Section fits the channel variations over the transmission interval. The received signal $r(t)$ is correlated with a locally generated PN sequence $g_{E}(t)$, over a bit transmission interval

$$
c\left(\tau+n T_{b}\right)=\frac{1}{T_{b}} \int_{\tau+n T_{b}}^{\tau+(n+1) T_{b}} r(t) g_{E}(t-\tau) d t
$$

where $\tau \varepsilon\left[0, T_{b}\right]$. 


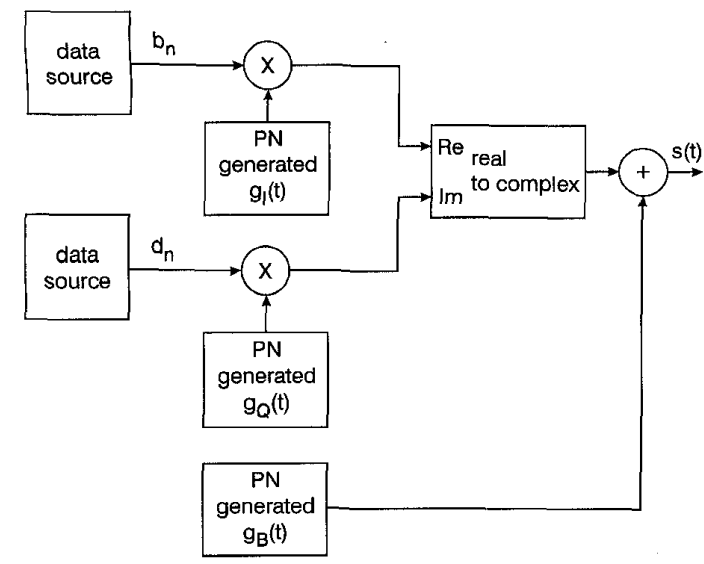

Fig.2 The transmitter model

Assuming a slow fading, we have parameters $\alpha_{i}$ and $\vartheta_{i}$ constant over at least two consecutive bits. Then eqn. 14, written as a function of the variable $t$, is

$$
\begin{aligned}
c(t)= & \sum_{n=0}^{+\infty} \sum_{i=1}^{L}\left[\alpha_{i} e^{j \vartheta_{i}} \delta\left(t-\tau_{i}-n T_{b}\right)\right]+c_{E, E}(t) \\
& +c_{E, I}(t)+c_{E, Q}(t)+c_{E, n}(t)
\end{aligned}
$$

where:

$\delta\left(t-\tau_{i}-n T_{b}\right)=1$, if $t=\tau_{i}+n T_{b}$; or $=0$, otherwise

$c_{E, E}(t)=$ the contribution of the auto-correlation of $g_{E}(t)$ when $\tau \neq \tau_{i}$

$c_{E, I}(t)=$ the contribution of the cross-correlation between $g_{E}(t)$ and $g_{I}(t)$

$c_{E, Q}(t)=$ the contribution of the cross-correlation between $g_{E}(t)$ and $g_{Q}(t)$

$c_{E, n}(t)=$ the contribution of the cross-correlation between $g_{E}(t)$ and $n(t)$

Assuming as negligible the contribution of the terms $c_{E, E}(t), c_{E, X}(t), c_{E Q}(t)$ (perfect orthogonal PN sequences) and $c_{E, n}(t)$ we have

$$
c(t) \cong \sum_{n=0}^{+\infty} \sum_{i=1}^{L} \alpha_{i} e^{j \vartheta_{i}} \delta\left(t-\tau_{i}-n T_{b}\right)
$$

Furthermore, the sampling frequency is chosen so that

$$
\left|\tau_{i}-\tau_{j}\right|>T_{c} \quad \forall i \neq j
$$

where $\tau_{i}$ and $\tau_{j}$ are the time delays introduced by the $i$ th path and the $j$ th path, respectively. This is related to the Rayleigh faded paths resolvability at the receiver side. The channel parameters estimation is obtained considering the correlation function

$$
\begin{aligned}
D(t) & =(\operatorname{Re}\{c(t)\})^{2}+(\operatorname{Im}\{c(t)\})^{2} \\
& \cong \sum_{n=0}^{+\infty} \sum_{i=1}^{L} \alpha_{i}^{2} \delta\left(t-\tau_{i}-n T_{b}\right)
\end{aligned}
$$

A 3-D graphical representation of eqn. 18 can be obtained considering the surface formed with $n$ correlation functions, one for each transmitted bit (Fig. 3).

The delays associated to the $L$ paths, over the generic symbol $n$, are found by searching for the $t=\hat{\boldsymbol{\tau}}_{j}$ values, for $j=1, \ldots, L$ so that $D\left(\hat{\tau}_{j}\right)$ assumes maximum values in the interval $\left[n T_{b},(n+1) T_{b}\right]$.

The estimated phase shift introduced by the $j$ th path, on the symbol $n$, is derived by substituting the found value $\hat{\tau}_{j}$ in eqn. 16

$$
c\left(\hat{\tau}_{j}+n T_{b}\right)=\hat{\alpha}_{j} e^{\hat{\vartheta}_{j}}
$$

After the channel estimation, a detection scheme based on the RAKE approach (Fig. 4), can be implemented. For each RAKE arm and for a fixed data symbol $n$ the following correlations are performed

$$
\begin{gathered}
c_{I}\left(\hat{\tau}_{j}+n T_{b}\right)=\frac{1}{T_{b}} \int_{\hat{\tau}_{j}+n T_{b}}^{\hat{\tau}_{j}+(n+1) T_{b}} r(t) g_{I}\left(t-\hat{\tau}_{j}-n T_{b}\right) d t \\
j=1, \ldots, L \quad(20) \\
c_{Q}\left(\hat{\tau}_{j}+n T_{b}\right)=\frac{1}{T_{b}} \int_{\hat{\tau}_{j}+n T_{b}}^{\hat{\tau}_{j}+(n+1) T_{b}} r(t) g_{Q}\left(t-\hat{\tau}_{j}-n T_{b}\right) d t \\
j=1, \ldots, L \quad(21)
\end{gathered}
$$

Eqns. 20 and 21 contain the informative components associated with the $j$ th path affected by a unknown phase-shift $\vartheta_{j}$ introduced by the communication channel. These components can be retrieved combining eqns. 20 and 21 with eqn. 19.

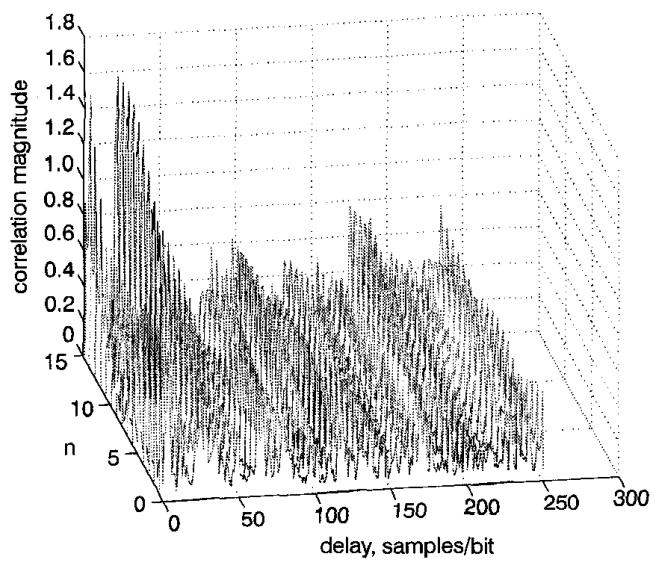

Fig.3 The correlation function

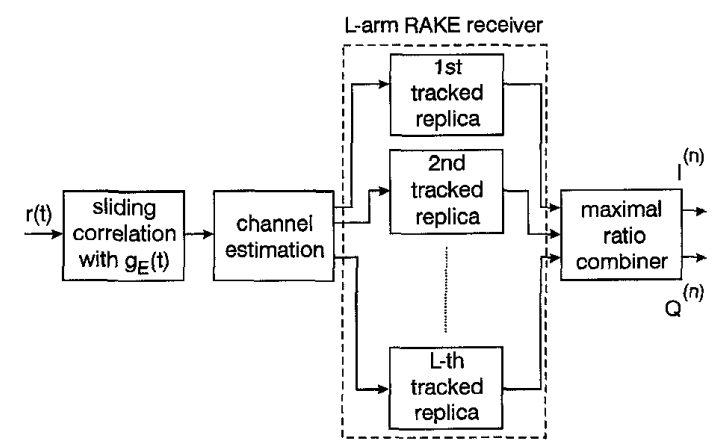

Fig.4 The receiver scheme

Hence the RAKE receiver outcome is

$$
\begin{gathered}
I_{j}^{(n)}=c_{I}\left(\hat{\tau}_{j}+n T_{b}\right) \cdot c^{*}\left(\hat{\tau}_{j}+n T_{b}\right) \\
Q_{j}^{(n)}=c_{Q}\left(\hat{\tau}_{j}+n T_{b}\right) \cdot c^{*}\left(\hat{\tau}_{j}+n T_{b}\right)
\end{gathered}
$$

where $I_{j}^{(n)} Q_{j}^{(n)}$ are, respectively, the in-phase and quadrature data components coming from the $j$ th path. The 
two decision variables are obtained as

$$
\begin{aligned}
I^{(n)} & =\sum_{j=1}^{L} I_{j}^{(n)} \\
Q^{(n)} & =\sum_{j=1}^{L} Q_{j}^{(n)}
\end{aligned}
$$

which are identical to the output of a maximum ratio combiner with an $L$ th order of diversity [14].

\section{Performance evaluation}

This Section deals with the simulation results of the digital receiver discussed in the previous Section. Owing to the difficulties in an analytical approach to this task, we have resorted to computer simulation. The communication channel has been modelled according to the GSM recommendation 5.05 (urban area) [13]. In particular, we have assumed $L$ equal to six. Parameters $\tau_{k}$, for $1 \leq k \leq L$, have been considered fixed, with values given in Table 1. Conversely, parameters $\alpha_{k}, \vartheta_{k}$, for $1 \leq k \leq L$, have been considered as statistically independent and identically distributed random variables. As stated before, independent uniform probability distributions on $[0,2 \pi)$ have been assumed for $\vartheta_{k}, 1 \leq k \leq$ $L$, while the random variables $\alpha_{k}, 1 \leq k \leq L$, have been characterised statistically by independent Rayleigh distributions with mean values given in Table 1 [13]. Each of the in-phase and quadrature informative bits $b_{n}$ and $d_{n}$, are spread with a sequence set of 127 preferentially phased Gold codes [14] operating at a rate of 6.5 Mchip/s.

Table 1: Channel parameter values

\begin{tabular}{lll}
\hline Path & Delay, $\mu \mathrm{s}$ & Attenuation, $\mathrm{dB}$ \\
\hline 1 & 0.0 & -3.0 \\
2 & 0.2 & 0.0 \\
3 & 0.5 & -2.0 \\
4 & 1.6 & -6.0 \\
5 & 2.3 & -8.0 \\
6 & 5.0 & -10.0 \\
\hline
\end{tabular}

It should be pointed out that at, this transmission rate, all the paths planned in the channel model are resolved. Moreover, the use of Gold PN sequences violates the property of perfect orthogonality among the PN sequences transmitted. In addition to this, the additive white Gaussian noise introduced by the downlink channel is not negligible in any practical application.

We prove shortly (Fig. 5) that it is convenient to perform the bit decision by means of a RAKE receiver based only on the signal components coming from the two most powerful paths. The bit-error probability of the proposed RAKE has been evaluated as a function of the signal-to-noise ratio $\bar{\gamma}_{b}$ at the receiver side, defined as

$$
\bar{\gamma}_{b}=\frac{E_{b}}{N_{0}} \sum_{j=1}^{L} \overline{\alpha_{j}^{2}}=\sum_{j=1}^{L} \bar{\gamma}_{j}
$$

where $E_{b}$ is the bit energy, $N_{0}$ is the one side noise power density and $\bar{\alpha}_{j}^{2}$ is the mean squared value of the random variable $\alpha_{j}$. Each of the $\left\{g_{j}\right\}$ is distributed according to a chi-squared distribution with 2 degrees of freedom

$$
p\left(\gamma_{j}\right)=\frac{1}{\bar{\gamma}_{j}} e^{-\frac{\gamma_{j}}{\bar{\gamma}_{j}}}
$$

where $\bar{\gamma}_{j}$ is the average SNR for the $j$ th path, defined as

$$
\bar{\gamma}_{j}=\frac{E_{b}}{N_{0}} \overline{\alpha_{j}^{2}}
$$

The performance of the proposed RAKE receiver will be compared with that of an ideal RAKE receiver, i.e. a RAKE receiver based on a perfect knowledge of the channel parameters and assuming a perfect orthogonality of the PN sequences. The bit-error probability for such an ideal RAKE receiver has been derived in [14] as

$$
P_{e, i d}=\frac{1}{2} \sum_{j=1}^{L} \pi_{j}\left[1-\sqrt{\frac{\bar{\gamma}_{j}\left(1-\rho_{r}\right)}{2+\bar{\gamma}_{j}\left(1-\rho_{r}\right)}}\right]
$$

where $\rho_{r}$ is the cross-correlator coefficient [14] and $\pi_{j}$ is defined as

$$
\pi_{j}=\prod_{\substack{i=1 \\ i \neq j}}^{L} \frac{\bar{\gamma}_{j}}{\bar{\gamma}_{j}+\bar{\gamma}_{i}}
$$

Under the assumption of $\rho_{r}=-1$ and $\bar{\gamma}_{k}>1$ eqn. 30 can be approximated as

$$
P_{e, i d} \cong\left(\begin{array}{c}
2 L-1 \\
L
\end{array}\right) \prod_{j=1}^{L} \frac{1}{4 \bar{\gamma}_{j}}
$$

Fig. 5 shows the bit-error probability $\left(P_{e}\right)$ obtained by means of the proposed RAKE receiver in comparison with $P_{e, i d}$. The results shown in this Figure have been derived under the assumption of only one active user and a Doppler spread $\left(f_{D}\right)$ equal to $10 \mathrm{~Hz}$. Fig. 5 highlights the good behaviour of the proposed RAKE receiver, also in comparison with the ideal RAKE receiver.

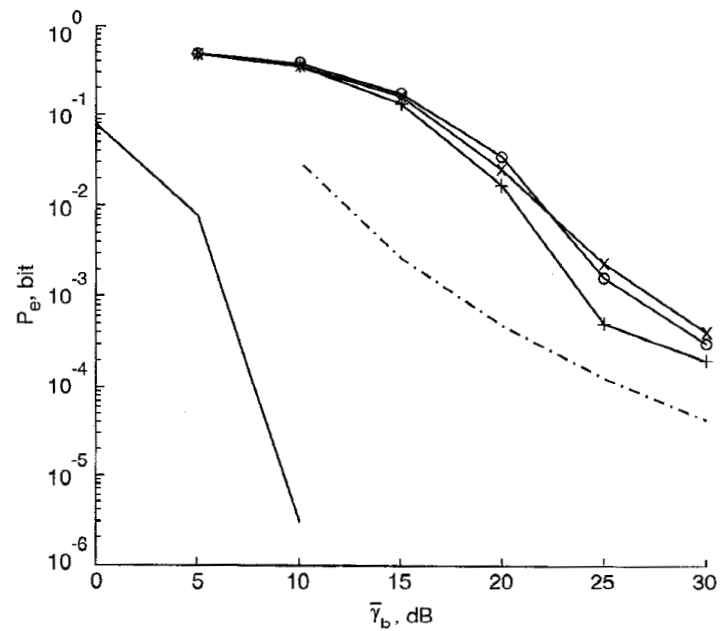

Fig.5 Bit-error rate comparisons, $K=1, f_{D}=10 \mathrm{~Hz}$ (slow fading) -1 AWGN (no fading)
-- one-path estimate
$-\times-$ three-path estimate
$----P_{e, i d}$ (ideal case)
-+- two-path estimate

It is also evident in Fig. 5 that the best performance for the proposed RAKE receiver is attained by processing the signal replicas coming from the two powerful paths. The reasons for this are: the use of PN sequences of the Gold type which violate the property 
of perfect orthogonality among sequences, and the presence of noise introduced by the downlink channel.
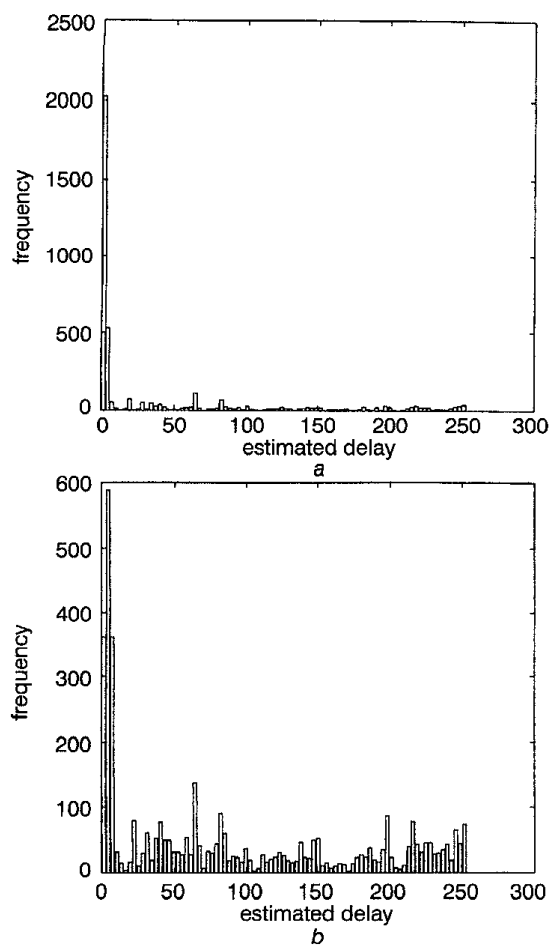

Fig.6 Histogram of the estimated delay for an infinite signal-to-noise ratio at the receiver side

$a$ Most powerful replica
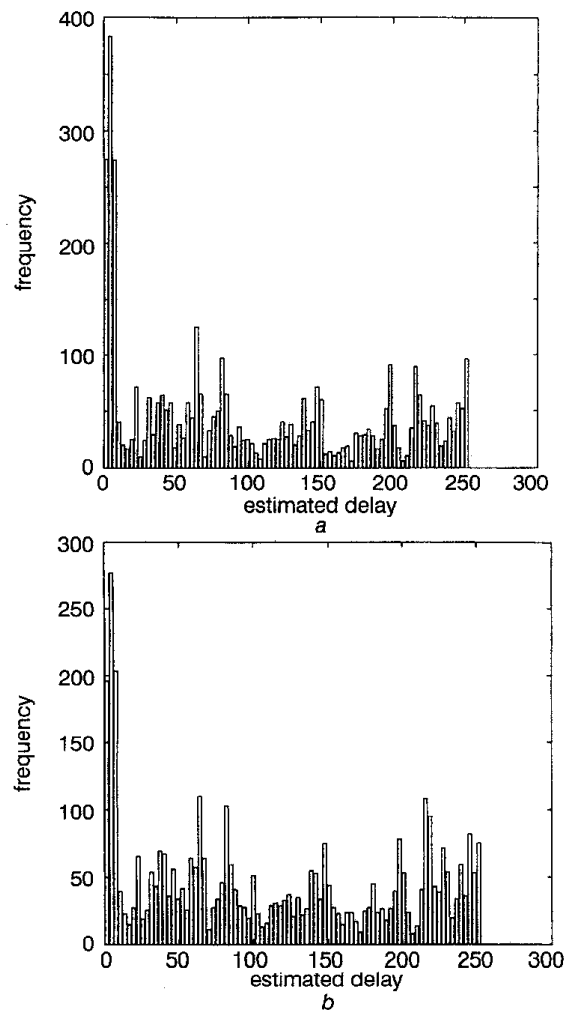

Fig.7 Histogram of the estimated delay for an infinite signal-to-noise ratio at the receiver side $a$ Third powerful replica

$b$ Fourth powerful replica
Hence the performance of the proposed RAKE receiver is influenced by the non-ideal behaviour of the channel parameters estimation technique outlined in the previous Section and by the channel noise.

The effects of non-perfect channel parameter estimation are outlined in Figs. 6 and 7. The results shown in these Figures have been obtained by carrying out a transmission of about 2000 informative bits. The delays introduced by the first and the second paths (first two most powerful replicas) are recognised with high reliability, conversely the delays associated with the third and fourth paths are estimated with a low reliability and are mingled with the first two paths.

Fig. 8 shows the bit-error probability as a function of the number of simultaneous users $(K)$. This Figure highlights again that the best performance can be achieved by processing only the signal components associated with the two most powerful replicas.

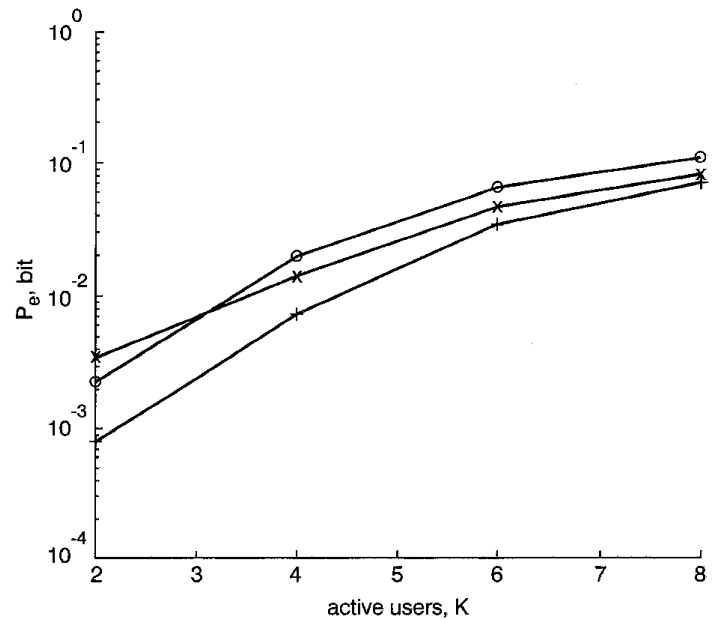

Fig. 8 Bit error rate comparisons, $f_{D}=10 \mathrm{~Hz}$

- 0 AWGN (no fading)

$-x-$ three-path estimate

- + - two-path estimate

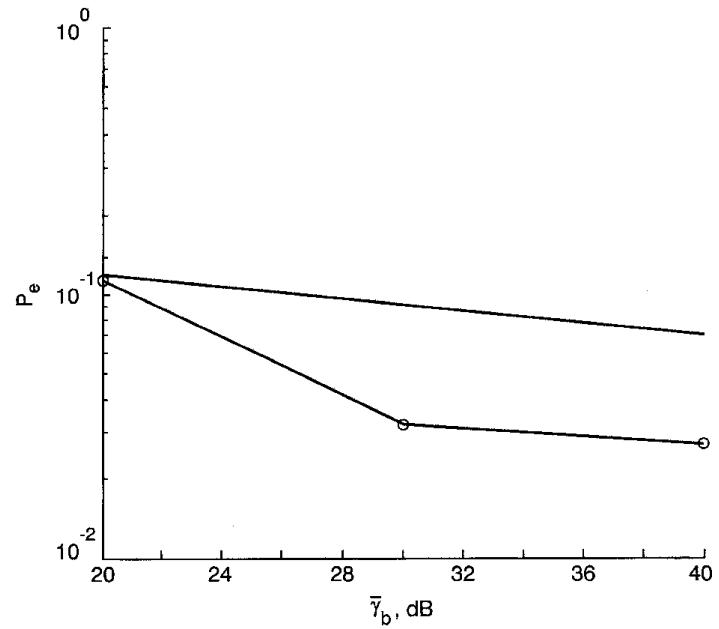

Fig.9 Bit error rate comparisons, $K=10, f_{D}=10 \mathrm{~Hz}$

- - proposed RAKE receiver (two-path estimate) ideal rejection receiver (QPSK)

In Fig. 9 the performance of the proposed RAKE receiver is compared with that obtained by an ideal rejection receiver using a QPSK modulation scheme 
(i.e. a RAKE receiver which ideally tracks and processes only the first received replica of the transmitted signal). The best behaviour of the proposed Rake receiver is again evident in the Figure.

The results presented in this Section have been limited to the case of $f_{D}$ equal to $10 \mathrm{~Hz}$. However, the proposed RAKE receiver exhibits good behaviour even at higher values of $f_{D}$, on the condition that the basic assumption of considering the channel parameters constant over at least two bit intervals is fulfilled. Moreover, it is important to emphasise that the performance of the proposed RAKE receiver (as the classical one) is strongly influenced by the multiple access interference (MAI) level. In particular, this cuts off the chance of increasing the channel parameter estimates by the transmission of the pilot signal at a higher power than that devoted to the information bearing signals. In order to relax the growth of the MAI level, suitable approaches should be used. However, this topic requires an in depth investigation which has been left to a future study.

\section{Conclusions}

The downlink performance of a practical RAKE receiver in a DS-CDMA mobile communication system has been analysed in a typical multipath fading environment. Channel parameter estimation has been accomplished at each receiving by means of a sliding correlation performed on a pilot signal broadcast by the base station. A performance evaluation, obtained by means of computer simulations, has highlighted the good behaviour of a RAKE receiver which estimates communication channel parameters and tracks the signal coming from the two most powerful paths. It has been verified that the proposed RAKE receiver outperforms an ideal multipath rejection receiver which processes only the first received replica and also exhibits a favourable comparison with an ideal RAKE receiver.

\section{Acknowledgment}

This work was carried out under the financial support of MURST

\section{References}

1 KLEIN, A., and BAIER, A.W.: 'Linear unbiased data estimation in mobile radio system applying CDMA', IEEE J. Sel. Areas Commun., 1993, 11, (7), pp. 1058-1066

2 GILHOUSEN, K.S., JACOBS, I.M., PADOVANI, R., VITERBI, A., WEAVER, L.A., and WHEATLY, C.E.: 'On the capacity of cellular CDMA system', IEEE Trans. Veh. Technol., 1991, 5, pp. 303-312

3 SALMASI, A., and GILHOUSEN, K.S.: 'On the system design aspects of a code division multiple access (CDMA) applied to digital cellular and personal communication networks'. Presented at IEEE conference on Vehical technology, St. Louis, MO, USA, May 1991

4 OCHSNER, H.: "Analysis of pseudonoise spread-spectrum communication over randomly fading channels without assuming perfect synchronization'. Proceedings of Military commun. conference, MILCOM'82, Boston, MA, USA, Oct. 1982, pp. 8.3$1-8.3-5$

5 KCHAO, C., and STÜBER, G.L.: 'Analysis of a direct-sequence spread-spectrum cellular radio system', IEEE Trans. Commun. 1993, 41, (10), pp. 1507-1516

6 PRICE, R., and GREEN, P.E.: 'A communication technique for multipath channel', Proc. IRE, 1958, 46, (3), pp. 555-570

7 BRENNAN, D.G.: 'Linear diversity combining techniques', Proc. IRE, 1959, 47, (6), pp. 1075-1102

8 BELLO, P-A.: "Characterisation of randomly time-variant channels', IEEE Trans. Commun., 1983, 11, (12), pp. 360-393

9 LEE, W.C.Y.: 'Mobile communications design fundamentals' (Howard W. Sams, New York, 1986)

10 Proposal on channel transfer functions to be used in GSM tests late 1986, COST 207 TD, 86, 51-REV 3, WG1, Paris, France, 1986

11 OCHSNER, $\mathrm{H}$ : 'Direct-sequence spread-spectrum receiver for communication on frequency-selective fading channels', IEEE $J$ Sel. Areas Commun., 1987, 5, (2), pp. 188-193

12 VITERBI, A.J.: 'CDMA: principles of spread spectrum communications' (Addison Wesley, New York, 1995)

13 GSM Recommendation 05.05, Transmission and reception

14 PROAKIS, J.G.: ‘Digital communications' (McGraw-Hill, New York, 1995, 3rd edn.) 